\title{
A INTERDISCIPLINARIDADE EM CURSOS DE ENGENHARIA
}

\author{
Elisangela Pavanelo ${ }^{1}$ \\ José Silvério Edmundo Germano² \\ Priscila Lourenço Freitas-Lemes ${ }^{3}$
}

\section{RESUMO}

Mudanças na sociedade atual têm estimulado reflexões sobre qual seria o papel da universidade. Desse modo, no lugar da "hiperespecialização" das disciplinas no contexto universitário, tem-se a ideia da interdisciplinaridade como uma maneira de a educação ser entendida e trabalhada. Apesar da interdisciplinaridade nos cursos de Engenharia se caracterizar como um passo crucial para a formação de profissionais mais qualificados e preparados para o atual mercado de trabalho, as ações com esse enfoque ainda são poucas e isoladas. Este artigo visa apresentar a análise de um projeto interdisciplinar desenvolvido com alunos do primeiro ano do Curso Fundamental de Engenharia, envolvendo as disciplinas de Desenho Assistido por Computador, Laboratório de Física e Projeto e Construção de Aeromodelo. Os resultados vão desde o empenho dos alunos até as avaliações positivas das disciplinas, indicando que esse é, possivelmente, um caminho produtivo para os futuros profissionais da área.

Palavras-chave: Cursos de Engenharia. Interdisciplinaridade. Laboratório de Física.

\footnotetext{
${ }^{1}$ Universidade Estadual Paulista Júlio de Mesquita Filho, Campus Guaratinguetá, SP, Brasil.

${ }^{2}$ Instituto Tecnológico de Aeronáutica, São José dos Campos, SP, Brasil.

${ }^{3}$ Instituto Tecnológico de Aeronáutica e Universidade do Vale do Paraíba, São José dos Campos, SP, Brasil.
} 


\title{
THE INTERDISCIPLINARITY OF ENGINEERING COURSES
}

Elisangela Pavanelo

José Silvério Edmundo Germano

Priscila Lourenço Freitas-Lemes

\begin{abstract}
Changes in the present day society have stimulated reflections about what would be the role of the University. Thus, instead of "hyper specialization" of the disciplines in the university context, one has the idea of interdisciplinarity as a way of understanding and working on education. Despite of interdisciplinarity in engineering courses being characterized as a crucial step to better qualify and train professionals for the current job market, the actions with this emphasis are few and isolated. This article presents an interdisciplinary work developed for first year students of Fundamental Engineering Course, involving the disciplines of Technical Drawing, Computer Assisted Design, Physics and Aeronautics. The results of this work range from the rewarding commitment of the students to the positive evaluation of the disciplines, indicating that this is possibly a productive path for future professionals.
\end{abstract}

Keywords: Engineering courses. Interdisciplinary. Physics lab. 


\section{INTRODUÇÃO}

A sociedade vive nos últimos anos, de acordo com Silva (2009), uma revolução de proporções comparáveis às da Revolução Industrial. Essa nova sociedade que se apresenta é a do conhecimento e da informação, na qual, sugere o autor, a ciência e a tecnologia são a chave para o desenvolvimento econômico.

As mudanças na sociedade estimulam reflexões sobre qual seria o papel da universidade. A função dessa instituição não pode mais se concentrar em habilitar os acadêmicos para as especializações tradicionais ou apenas visar aos novos postos de trabalho que surgem. Esse papel hoje deve englobar uma formação de consciência e cidadania, estimulando o desenvolvimento de um profissional com novas características, dentre as quais se destacam a autonomia, o senso crítico e o desenvolvimento intelectual.

Em décadas passadas, a qualificação profissional adquirida por meio da educação era um critério qualificador de competição no mercado de trabalho. Atualmente, um profissional de sucesso é aquele que possui visão global da área em que atua, predisposição para o trabalho em equipe, conhecimento de mercado, iniciativa, espírito empreendedor, criatividade, dentre outros. E não mais aquele profissional que possui somente um conhecimento específico em dado assunto.

Em contraponto a esse movimento, temos como base em nossas universidades um currículo tradicional apoiado no que Morin (2005) chama de "hiperespecialização". Para ele, existe hoje a necessidade de uma reforma radical do modelo de ensino nas universidades e escolas, salientando a necessidade de passar da atual hiperespecialização para uma aprendizagem focada em trabalhos e que integre as várias áreas do conhecimento. Segundo o autor, apenas com essa mudança de paradigma no ensino as pessoas serão "capazes de compreender e enfrentar os problemas fundamentais da humanidade, cada vez mais complexos e globais" (MORIN, 2005, p. 11).

De acordo com Libâneo,

Torna-se necessário prover as condições para se obter mais qualidade de ensino, dentro de práticas participativas e colaborativas em que os docentes sejam protagonistas dos processos de mudança. A ideia é introduzir nos cursos espaços de reflexão conjunta, trocas de 
experiência, formas de negociação e tomada de decisões coletivas (LIBÂNEO, 2003, p. 2).

Libâneo (2003) também revela em seu trabalho que o alvo de muitas críticas dos alunos não é diretamente o professor, mas, sim, o ensino tradicional, isto é, um sistema de relações centrado apenas na didática da transmissão de informação, que reduz o estudante a um sujeito que recebe passivamente essa informação. Essa maneira de conduzir a aula na universidade não ajuda o aluno a conquistar, com os próprios recursos intelectuais e afetivos, uma sólida aprendizagem de conhecimentos, habilidades, valores, que é, segundo o autor, o aspecto mais relevante do ensinar.

Apresenta-se então, resumidamente, as características apontadas por Libâneo (2003) atribuídas à prática docente e ao processo de aprendizagem:

1. O foco, o nuclear da prática docente é a aprendizagem do aluno, resultante da sua própria atividade intelectual e prática realizada em parceria com os professores e colegas;

2. A sala de aula é um espaço de construção conjunta do conhecimento. É o lugar onde professores e alunos buscam juntos o conhecimento, estabelecem interações, diálogos, trocas;

3. A aprendizagem está relacionada com a atividade de pesquisa tanto do aluno quanto do professor. Implica promover situações em que o aluno aprenda a buscar informações, aprenda a localizá-las, analisá-las, relacioná-las com conhecimentos anteriores, dando-lhes significado próprio, a redigir conclusões, a observar situações de campo e registrálas, a buscar solução de problemas, dentre outros;

4. A sala de aula universitária hoje não pode mais ser entendida meramente como espaço físico em um tempo determinado (por exemplo, 2 horas) em que o professor transmite conhecimentos aos alunos. A sala de aula é todo espaço em que os alunos podem aprender;

5. Toda aprendizagem precisa ser significativa, isto é, os conteúdos precisam fazer sentido para o aluno, com base nos próprios sentidos que os alunos atribuem ao que estão aprendendo;

6. A sala de aula implica uma aproximação entre a teoria e a prática;

7. A aprendizagem universitária está associada ao aprender a pensar e ao aprender a aprender (LIBÂNEO, 2003, p. 8). 


\section{METODOLOGIAS ATIVAS}

Ao trabalharmos com o conceito de interdisciplinaridade, entendemos ser necessária uma metodologia que supra todas as necessidades e que ocorra em sala de aula ou mesmo nos laboratórios utilizados.

As metodologias ativas podem auxiliar o professor a suprir essa necessidade, sendo esse um termo utilizado para um conjunto de práticas pedagógicas que incentivam a interação em sala de aula (MORAN, 2015). Nelas, o aluno não é apenas um receptor do conteúdo, ele, de alguma forma, é estimulado a também ser responsável pela construção do próprio conhecimento.

Na educação presencial, de acordo com Moran (2015), ensinamos com materiais e comunicações escritos, orais e audiovisuais, previamente selecionados ou elaborados. 0 autor destaca que esses são extremamente importantes, mas que a melhor forma de fazer o aluno aprender é combinar de maneira equilibrada atividades, desafios e informação contextualizada.

Moran (2015) até exemplifica com uma relação bem prática: "para aprender a dirigir um carro, não basta ler muito sobre esse tema; tem que experimentar, rodar com ele em diversas situações com supervisão, para depois poder assumir o comando do veículo sem riscos" (MORAN, 2015, p. 3).

Desse modo, o autor destaca que as metodologias precisam acompanhar os objetivos pretendidos pelo professor, pois, se o que se quer são alunos proativos, precisa-se adotar metodologias em que eles se envolvam em atividades cada vez mais complexas, em que tenham que tomar decisões e avaliar os resultados, com apoio de materiais relevantes.

Se o que se quer são alunos criativos, eles precisam experimentar inúmeras novas possibilidades de mostrar iniciativa.

De acordo com Valente (2013),

em oposição à aprendizagem passiva, bancária, baseada na transmissão de informação, o aluno assume uma postura mais ativa, na qual ele resolve problemas, desenvolve projetos e, com isto, cria oportunidades para a construção de conhecimento. Diversas estratégias têm sido utilizadas para promover a aprendizagem ativa 
como a aprendizagem baseada na pesquisa, o uso de jogos ou o problem based learning (PBL) (VALENTE, 2013, p. 1).

Moran (2015) destaca que os desafios bem planejados podem contribuir para mobilizar as competências desejadas, intelectuais, emocionais, pessoais e comunicacionais, exigindo que o aluno pesquise, avalie situações, pontos de vista diferentes, faça escolhas, assuma riscos, aprenda pela descoberta.

\section{SOBRE A INTERDISCIPLINARIDADE}

Existe uma necessidade atual de compreensão do conhecimento de forma global e abrangente, em que apenas a contextualização de certo conteúdo torna-se algo artificial, e a interdisciplinaridade não é alcançada. Com isso, surgem dificuldades de compreensão dos conceitos em aplicação real, e o conteúdo escolar acaba por não fazer sentido para o aluno.

De acordo com D’Ambrósio (2013),

EDUCAÇÃO é uma estratégia desenvolvida pelas sociedades para:

(I) estimular e possibilitar que cada indivíduo atinja seu potencial criativo, e

(II) preparar indivíduos para a ação comum, com vistas a viver em sociedade, praticando a cidadania plena (D’AMBRÓsıO, 2013, p. 3).

Para esse autor, o maior problema da educação é a maneira de se encarar o conhecimento, a maneira de se ver as disciplinas que, com raras exceções, apresentam programas que têm como base conteúdos que são "Desinteressantes, Obsoletos e Inúteis" (D’AMBRÓsIO, 2013, p. 7). Segundo ele, nesse caso, o programa "D O I" para ser desenvolvido na integralidade. D’Ambrósio (2013) ainda ressalta que insistir no desinteressante, obsoleto e inútil esgota o tempo e a energia do aluno e do professor, dói para ambos e prejudica o aprendizado de coisas que são interessantes, modernas e úteis, e que são essenciais para se viver na sociedade moderna.

Desse modo, D’Ambrósio (2013) afirma que o desafio está sintetizado no trinômio: porque ensinar; o que ensinar; como ensinar. E tendo como princípio básico ancorar a prática educativa nos objetivos maiores da educação, enfatizando conhecimentos e comportamentos relevantes nos mundos atual e futuro. 
Em uma perspectiva interdisciplinar, o aluno torna-se comprometido, responsável e incentivado a interagir com o meio em que está, cabendo ao professor proporcionar situações que o auxilie a ser sujeito da própria aprendizagem.

A partir de então, Luck (2001) evidencia que:

A interdisciplinaridade é o processo de integração e engajamento de educadores, num trabalho conjunto, de interação das disciplinas do currículo escolar entre si e com a realidade, de modo a superar a fragmentalização do ensino, objetivando a formação integral dos alunos, a fim de que exerçam a cidadania, mediante uma visão global de mundo e com capacidade para enfrentar os problemas complexos, amplos e globais da realidade (LUCK, 2001, p. 64).

Acredita-se, desse modo, como aponta Peleias et al. (2011), que os estudos e pesquisas sobre interdisciplinaridade podem contribuir de maneira contundente, fomentando propostas e posturas metodológicas que atendam às reais necessidades dos alunos, permitindo aprendizagens significativas no contexto universitário.

\section{A INTERDISCIPLINARIDADE E O ENSINO DE ENGENHARIA}

Interdisciplinaridade é um tema que se discute desde a década de 1970, por diversos educadores no Brasil, tendo como objetivo superar a dissociação dos saberes entre os especialistas e, desse modo, procurando atuar no espaço de negação e superação de fronteiras disciplinares.

Loder (2002) aponta que a visão contemporânea da engenharia é a de uma profissão que se impõe para resolver problemas tecnológicos, alavancando o progresso da sociedade, ora atuando como tecnologia ora como ciência, regida principalmente pelos impactos sociais, ambientais, econômicos e técnicos. Nessa perspectiva, a educação em engenharia necessita de mudanças, adotando, cada vez mais, diretrizes que privilegiem a formação do cidadão -engenheiro em lugar do técnico-engenheiro.

Nesse sentido, Maines (2001) apresenta as seguintes questões:

O que pode ser feito para formar acadêmicos de engenharia para que estes tragam em sua complexa bagagem formativa, algo a mais que simples acúmulo de saberes técnicos, ou habilidades para trabalhar em seu cotidiano profissional? O que pode ser feito para 
facilitar/estimular atitudes interdisciplinares no futuro profissional de engenharia? (MAINES, 2001, p. 42).

Pode-se perceber que atitudes interdisciplinares nos cursos de Engenharia caracterizam-se como um passo crucial para a formação de profissionais mais qualificados e preparados para o atual mercado de trabalho, tornando essa formação mais completa e integrada, associando o saber oriundo de diversas áreas.

Em pesquisas como as de Schor e Demajorovic (2002), Queiroz et al. (2012), Maines (2001), aponta-se para a dificuldade do trabalho interdisciplinar em cursos de Engenharia, seja pela complicada relação entre a teoria e a prática, seja porque os professores necessitam conhecer não só sobre a área em que atuam, mas também sobre as outras envolvidas, seja pelas diferenças entre as distintas áreas, em linguagem, expressão, ritmo de pesquisa, dentre outros. Desse modo, práticas com essa natureza ainda são pouco comuns.

Maines (2001) ressalta a importância, para o ensino de Engenharia, de ações que estabeleçam relações entre as diferentes disciplinas e que tais ações são extremamente necessárias e bemvindas, sendo esse um primeiro passo necessário para que se possa, futuramente, concretizar o processo de ensino e de aprendizagem dentro de uma realidade interdisciplinar.

\section{UM CAMINHO PARA O ENSINO INTERDISCIPLINAR EM CURSOS DE ENGENHARIA}

De acordo com Andrade, Lopes e Carvalho (2009), existe atualmente uma busca por uma educação científica que seja capaz de proporcionar o conhecimento do mundo natural como elemento para a compreensão do todo complexo denominado como cultura científica, e a experimentação é uma forma de favorecer o estabelecimento de um elo entre o mundo dos objetos, o mundo dos conceitos, leis e teorias, o mundo das linguagens simbólicas, além do papel importante que esta pode vir a desempenhar na formação do indivíduo.

Desse modo, Andrade, Lopes e Carvalho (2009) também destacam que o Laboratório de Física se caracteriza como uma ferramenta relevante no estabelecimento desse elo, por evidenciar a interação entre o sujeito e o objeto explorado e desses com o conhecimento científico e com a cultura científica. Nos cursos de Engenharia, o Laboratório de Física também possui esse enfoque. 
A partir da nossa experiência docente, percebemos que, apesar de a utilização do Laboratório de Física nos cursos de Engenharia ser feita com certa frequência, o que se pode verificar, muitas vezes, é que as atividades realizadas nesse ambiente buscam apenas desenvolver nos alunos habilidades práticas, como comprovação/verificação de leis e teorias que auxiliam na compreensão de conceitos científicos. Embora esses aspectos auxiliem na compreensão dos conceitos, transmitindo o conhecimento sobre o método científico, práticas dessa natureza podem não ser suficientes para explorar toda a potencialidade desse ambiente em um curso de Engenharia.

Com o objetivo de aproveitar esse ambiente de maneira que explorasse maiores possibilidades para o processo de ensino e aprendizagem, foi desenvolvido um projeto de interdisciplinaridade no Curso Fundamental de Engenharia, entre as disciplinas de Desenho Assistido por Computador, Laboratório de Física e Projeto e Construção de Aeromodelo, inicialmente no segundo semestre de 2014, repetindo-se no segundo semestre de 2015.

De acordo com Coimbra (2000), o trabalho interdisciplinar possui como característica ter um tema norteador, ou um objeto, em que duas ou mais disciplinas intencionalmente estabelecem conexões entre si para alcançar um conhecimento mais abrangente, "ao mesmo tempo diversificado e unificado" (COIMBRA, 2000, p. 58). Desse modo, é importante destacar que cada disciplina manteve a sua identidade e conservou a sua metodologia, porém, como o próprio Coimbra (2000) propõe, intercambiando hipóteses, elaborações e conclusões.

Coimbra ilustra esse trabalho da seguinte maneira:

Numa ação interdisciplinar as partes envolvidas dão-se as mãos, movimentam-se juntas como num balé, voltadas para o tema central. Aproximam-se, afastam-se; interpelam-se, respondem-se; ora se exibe o solista, ora se impõe o coro. O essencial da interdisciplinaridade consiste em produzir uma ação comum, mantendo cada participante o que Ihe é próprio (COIMBRA, 2000, p. 58).

O mesmo autor aponta ser imprescindível que as disciplinas envolvidas tentem trabalhar uma metodologia comum, que equalize as diferenças, transformando-as em semelhanças. Para tanto, propõe itens que poderiam ser apontados como necessários ao desenho dessa metodologia. Por exemplo: 
- situação da disciplina ou ciência no contexto do saber;

- definição do seu objetivo específico na construção do conhecimento;

- explicitação do seu método próprio, dos procedimentos peculiares;

- que relação tem ela com o objeto (ou projeto) em questão;

- que contribuições pode ela dar para o conhecimento interdisciplinar desejado;

- quais as adequações que seria necessário introduzir (COIMBRA, 2000, p. 64).

O trabalho interdisciplinar entre essas disciplinas foi realizado com o intuito de incentivar os alunos à reflexão sobre as novas competências e atitudes que permeiam, atualmente, o estudante de Engenharia e também a busca de novas alternativas de atividades pedagógicas que podem ser desenvolvidas no Laboratório de Física.

\section{APRESENTAÇÃO E DISCUSSÃO DOS RESULTADOS}

A proposta de projeto teve quatro meses de duração, que é o tempo relativo a um semestre letivo, envolvendo, em 2014, quando foi realizada a primeira prática, 180 alunos do 1 ano do Curso Fundamental de Engenharia, divididos em 56 grupos. Já na segunda prática, realizada em 2015, participaram 140 alunos, divididos em 50 grupos.

Torna-se necessário destacar algumas características dos alunos da instituição onde o projeto foi realizado. Eles estudam em tempo integral e moram no próprio campus. Dados do Exame Nacional de Desempenho de Estudantes (ENADE) de 2011 mostram que 60\% dos alunos se consideram brancos e $40 \%$, pardos. Com relação à renda familiar, $26 \%$ possuem renda ente dez e trinta salários mínimos e 15\%, acima de trinta. Sobre a escolarização dos pais, os dados apontam que $44 \%$ possuem curso superior. $E$, finalmente, $74 \%$ dos alunos cursaram o ensino médio em uma instituição particular. Esses alunos já ingressam no primeiro ano do curso com uma boa base de matemática.

Esses dados foram descritos com o objetivo de ilustrar que estamos desenvolvendo tal projeto no âmbito de uma instituição de ensino superior de Engenharia um pouco diferenciada da realidade da maioria do nosso país.

Vale destacar que, independentemente da metodologia adotada pelo professor da turma, todos os alunos são avaliados a partir de uma mesma prova, ao final de cada bimestre. 
Inicialmente, os grupos receberam um conjunto de peças (kit) de um aeromodelo TAYLORCRAFT EE-206. Cada kit é composto de asa, madeira para a fuselagem, leme de direção e estabilizador, cuja planta é apresentada a seguir. Em poder desses kits, os grupos tiveram, ao longo do semestre letivo, instruções e acompanhamento direto de professores da Divisão de Engenharia Aeronáutica sobre o processo de montagem e algumas instruções relativas aos componentes que fazem parte do aeromodelo. As atividades relativas à montagem dos aeromodelos foram realizadas pelos alunos, no Laboratório de Aeromodelismo da Divisão de Engenharia Aeronáutica.

Em paralelo, no Laboratório de Física, foi realizada a parte relativa à aprendizagem de todo o processo de metrologia, a fim de suportar as medições das peças envolvidas na montagem dos aeromodelos, ou seja, medição de todas as peças (comprimento, diâmetro, espessura, área, volume, dentre outras). Esse trabalho desenvolvido pelos alunos encontra-se ilustrado nas Figuras 1 e 2. Outra novidade que estamos introduzindo nos nossos laboratórios de Física é a utilização do software Mathematica ${ }^{\circledR}$, como ferramenta de suporte à análise e à estatística dos dados envolvidos nos processos de medição.

Figura 1 - Início da exploração do kit de montagem do avião pelos grupos

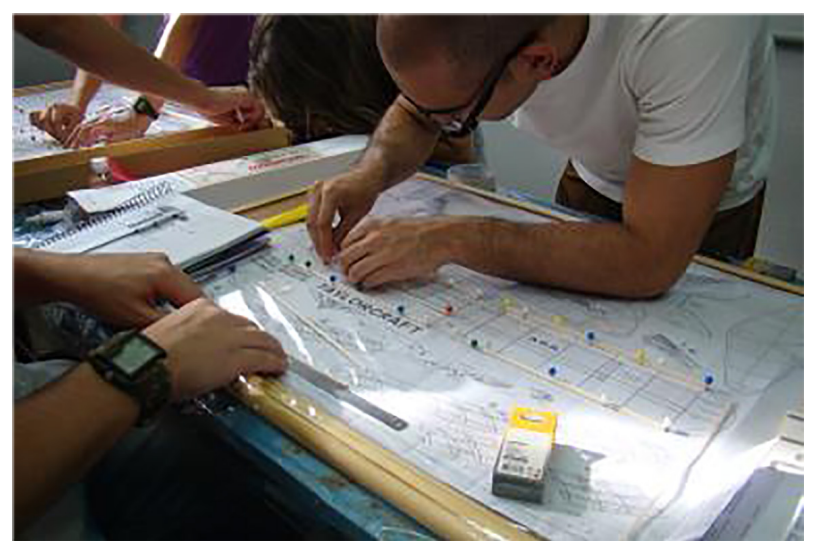


Figura 2 - Planta do aeromodelo entregue aos grupos em tamanho natural

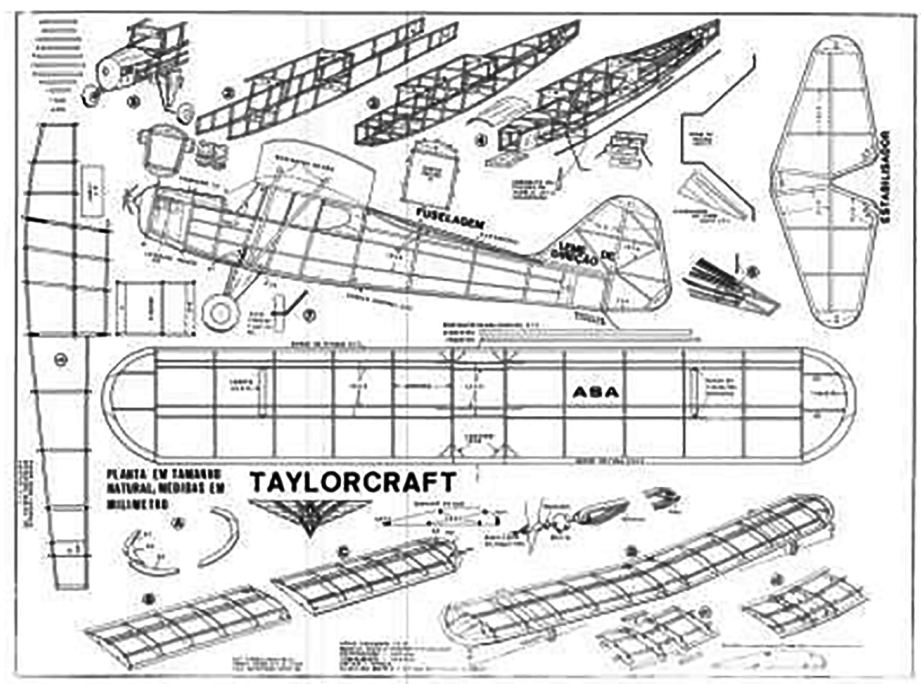

A disciplina de Desenho Assistido por Computador foi responsável pela construção virtual do aeromodelo que estava sendo feito ao longo do semestre. Esse tipo de abordagem num curso introdutório de desenho é interessante e motivadora, pois os alunos constroem peças que, no final, comporão e integrarão um sistema real, em vez de desenvolverem apenas peças aleatórias com o objetivo puramente de cumprir exercícios acadêmicos. A Figura 3 ilustra o resultado final de um dos grupos, nela podemos ver, à esquerda, o aeromodelo real construído e, à direita, a imagem do aeromodelo virtual desenvolvido na disciplina de Desenho Assistido por Computador. Já na Figura 4, os alunos de outro grupo exibem o aeromodelo virtual que fizeram, construído ao longo dessa disciplina.

No dia a dia das atividades dos alunos, eles usaram o Laboratório de Aeromodelismo do Curso de Engenharia Aeronáutica para a construção dos aeromodelos, sob orientação do professor da disciplina de Projeto e Construção de Aeromodelo.

Desse modo, a nossa proposta de interdisciplinaridade realizada no $1^{\circ}$ ano de Engenharia do Instituto Tecnológico da Aeronáutica (ITA) envolveu um número abrangente de profissionais de várias áreas do conhecimento, tais como: física básica, aeronáutica e desenho técnico. 
Figura 3 - Resultado final de um dos grupos. À esquerda, o aeromodelo real construído e, à direita, imagem do aeromodelo virtual, desenvolvido na disciplina de CAD (Computer-Aided Design ou Desenho Assistido por Computador)
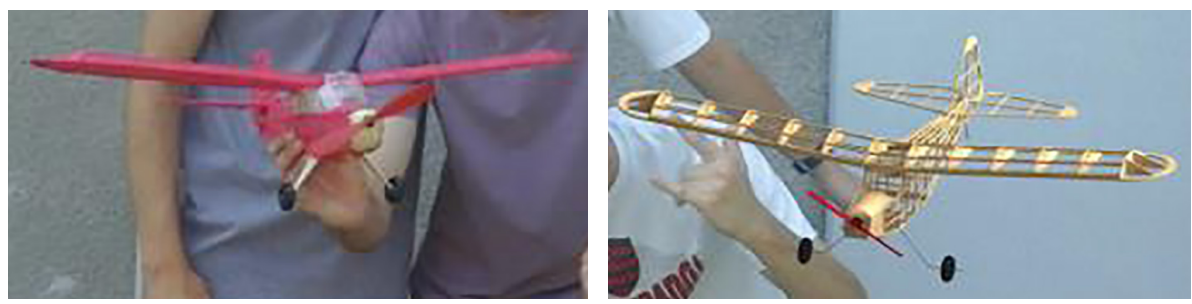

Figura 4-Resultado final de um dos grupos. Aeromodelo virtual, desenvolvido na disciplina de Desenho Assistido por Computador

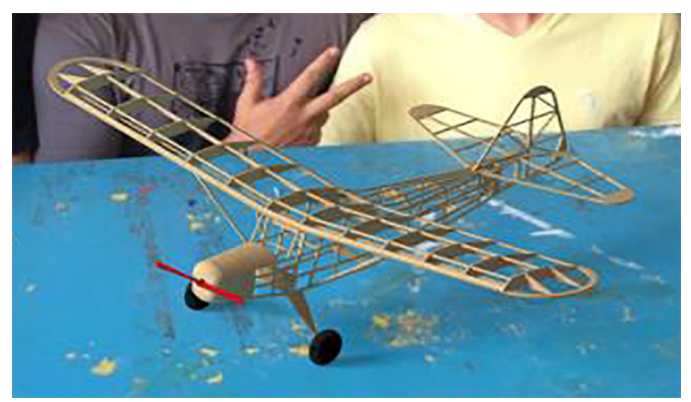

O trabalho interdisciplinar, nos dois semestres, foi desenvolvido de acordo com as etapas descritas a seguir.

Primeira etapa: montagem das ASAS do aeromodelo, antecedida de uma explicação sobre aerodinâmica, dada pelo professor da disciplina de Projeto e Construção de Aeromodelo, e a sustentação de uma aeronave.

Segunda etapa: montagem do LEME DE DIREÇÃO e do ESTABILIZADOR do aeromodelo, antecedida de uma explicação sobre a estabilidade e o controle de uma aeronave. 
Terceira etapa: montagem da FUSELAGEM do aeromodelo, antecedida de uma explicação sobre a estrutura de uma aeronave.

Quarta etapa: realização da ENTELAGEM do aeromodelo. As partes serão enteladas separadamente: asa, estabilizador, leme de direção e fuselagem.

Quinta etapa: integração das partes e cálculo do centro de gravidade (CG) e a compensação do voo planado do aeromodelo.

Com relação à avaliação, foram considerados, inicialmente, o desempenho e o envolvimento dos alunos nas atividades propostas e previstas nas etapas citadas do projeto, contemplando a frequência nas atividades didáticas e o desempenho do grupo, representando um percentual da nota da disciplina que fez parte do projeto. Além disso, os alunos realizaram avaliação teórica individual em cada disciplina, o que representou $30 \%$ da nota deles. E, com o objetivo de finalizar a avaliação, os alunos apresentam, no final do semestre, um relatório, em forma de artigo científico, a respeito dos resultados obtidos. Tal relatório possui a seguinte estrutura: resumo, introdução, fundamentação teórica, metodologia, resultados, conclusão e bibliografia. Desse modo, utilizamos esses instrumentos para avaliar o projeto interdisciplinar, ou seja, as avaliações realizadas individualmente e a qualidade do relatório final.

Durante o processo de construção do aeromodelo, muitos aspectos da Física, da Engenharia Aeronáutica e do uso de software de CAD podem ser explorados por todos os professores envolvidos, tais como: o cálculo da estimação do tensor de inércia, a determinação da polar de arrasto, o cálculo das derivadas de estabilidade e até mesmo a história da aviação, etc.

Um trecho do relatório de um dos grupos é destacado a seguir para ilustrar tal fato:

A montagem do aeromodelo físico é bastante artesanal e requer o uso de colas de diferentes densidades e propriedades para colar todas as peças. Além disso, quatro demãos de dope foram aplicadas em toda a aeronave para aumentar sua resistência estrutural, o que não são considerados no projeto virtual. Por isso, esperávamos que a massa total do aeromodelo fosse superior à do modelo virtual, o que de fato é. Entretanto, o que surpreende é que a massa do avião pronto é cerca de 2,6 vezes maior do que o modelo ideal projetado em computador. Isso significa que as substâncias adicionais influenciam consideravelmente na mecânica de voo da aeronave, o que deve ser considerado em futuras modelagens computacionais. Para o centro de gravidade, esperávamos um desvio pequeno entre o modelo real e virtual, 
porque a cola e o dope foram aplicados de maneira aproximadamente uniforme por toda aeronave. Todavia, constatamos um desvio relativo de cerca de $14,5 \% .^{4}$

Seria interessante que o grupo realizasse o cálculo da densidade da madeira balsa utilizada após a aplicação de quatro demãos de dope, com o objetivo de obter massas semelhantes para o modelo virtual e para o real. Esse procedimento também poderia aproximar os valores dos centros de gravidade entre os modelos.

\section{CONSIDERAÇÕES FINAIS}

De acordo com os pontos discutidos neste trabalho, os estudos e pesquisas sobre interdisciplinaridade podem contribuir de maneira contundente para o ensino, fomentando propostas metodológicas que viabilizem aprendizagens significativas no contexto universitário.

Além de incentivar os alunos a uma reflexão sobre as novas competências e atitudes que permeiam o profissional de Engenharia, a interdisciplinaridade entre as disciplinas de Desenho Assistido por Computador, Laboratório de Física I e Projeto e Construção de Aeromodelo ganharam significado concreto dentro do curso. Respeitando o escopo de cada disciplina, o desenvolvimento da interdisciplinaridade se tornou concreto no curso de Engenharia.

Entende-se que práticas como essa, ilustradas nos resultados apresentados na Figura 5, precisam ser incentivadas e exploradas nos cursos de Engenharia, nos quais os professores, técnicos e alunos trabalham em parceria para a formação de um profissional competente e preparado para os desafios de uma nova sociedade.

${ }^{4}$ Trecho do relatório produzido, como parte da avaliação, por um dos grupos participantes do projeto. 
Figura 5 - Voo do aeromodelo real de um dos grupos

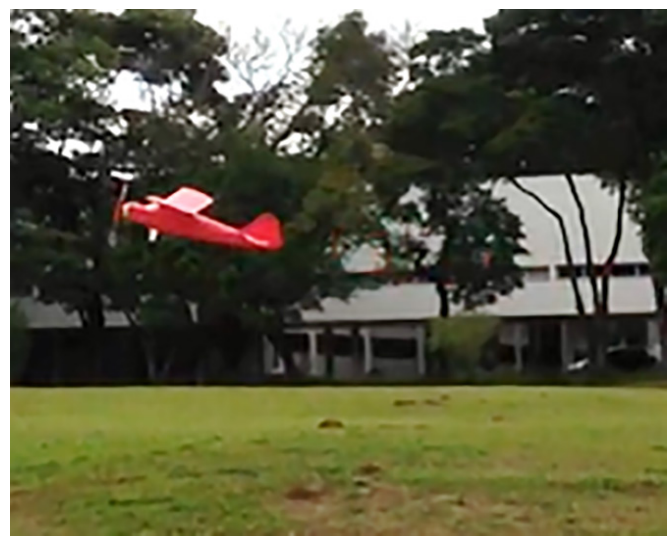

A construção do aeromodelo mostra o bom resultado de um projeto interdisciplinar que não prejudica o andamento de cada uma das disciplinas e contribui para fomentar discussões sobre o significado dos conteúdos e objetivos de cada uma.

A interdisciplinaridade, pensando na formação global do aluno, nos leva à compreensão, na prática, de que, para ser um bom engenheiro, não basta somente gostar de matemática ou física e, mesmo, dominar conhecimentos técnicos específicos. Para solucionar problemas do século XXI e produzir inovações tecnológicas, também são exigidas dos novos profissionais características diferenciais, como comunicação, facilidade para trabalhar em equipe, liderança, empreendedorismo e criatividade.

\section{AGRADECIMENTOS}

Priscila Lourenço Freitas-Lemes agradece à Coordenação de Aperfeiçoamento de Pessoal de Nível Superior (CAPES) pelo apoio financeiro. 


\section{REFERÊNCIAS}

ANDRADE, J. A. N.; LOPES, N. C.; CARVALHO, W. L. P. Uma análise crítica do laboratório didático de Física: a experimentação como uma ferramenta para a cultura científica. In: VII ENPEC - ENCONTRO NACIONAL DE PESQUISA EM EDUCAÇÃO EM CIÊNCIAS. Florianópolis, 2009.

COIMBRA, J. A. A. Considerações sobre a interdisciplinaridade. In: PHILIPPI JR., A. Interdisciplinaridade em ciências ambientais. São Paulo: Signus, 2000.

D’AMBRÓSIO, U. A interdisciplinaridade e a transdisciplinaridade na formação do professor. Palestra proferida na Universidade Bandeirante Anhanguera, Jornada de Educação, 2013. Disponível em: <https://docs.google.com/file/d/OB4JIJny_-_7pcjJlem9BZEhYNkE/edit>. Acesso em: 15 mar. 2017.

LIBÂNEO, J. C. O ensino de graduação na universidade: a aula universitária. Goiânia: UCG, 2003.

LODER, L. L. Epistemologia versus Pedagogia: o locus do professor de Engenharia. 2002, 115 f. Dissertação (Mestrado em Educação) - Universidade Federal do Rio Grande do Sul, Porto Alegre, 2002.

LUCK, H. Pedagogia da interdisciplinaridade. Fundamentos teórico-metodológicos. Petrópolis: Vozes, 2001.

MAINES, A. Interdisciplinaridade no ensino de Engenharia. In: XXIX CONGRESSO BRASILEIRO DE EDUCAÇÃO EM ENGENHARIA. Anais... Porto Alegre, 2001.

MORAN, J. Mudando a educação com metodologias ativas. In: SOUZA, Carlos Alberto; MORALES, Ofelia Elisa Torres (Org.). In: Coleção Mídias Contemporâneas. Convergências Midiáticas, Educação e Cidadania: aproximações jovens. v. II. Ponta Grossa: Foca FotoPROEX/UEPG, 2015.

MORIN, E. Educação e complexidade, os sete saberes e outros ensaios. São Paulo: Cortez, 2005.

PELEIAS, I. R.; MENDONÇA, J. F.; SLOMSKI, V. G.; FAZENDA, I. C. A. Interdisciplinaridade no Ensino Superior: Análise da percepção de professores de Controladoria em cursos de Ciências Contábeis na cidade de São Paulo. Avaliação, Campinas, v. 16, n. 3, nov. 2011.

QUEIROZ, M. L.; SANTOS, J. B.; OLIVEIRA, D. S.; RAMOS, S. C. J. R.; SILVA, L. S. C. Interdisciplinaridade no ensino de Engenharia: a experiência do $\mathrm{PET} /$ Observatório para 
o uso racional da água. In: XL CONGRESSO BRASILEIRO DE EDUCAÇÃO EM ENGENHARIA. Anais... Belém, 2012.

SILVA, Fabio Q. B. Cooperação empresa/universidade: contexto, análise e perspectivas. 2009a. Disponível em: <http://www.di.ufpe.br/ srlm/secomu96/fabio.htm>. Acesso em: 1 maio 2015.

SCHOR, T.; DEMAJOROVIC, J. Interdisciplinaridade em educação ambiental: utopia e prática. In: I ENCONTRO DA ASSOCIAÇÃO NACIONAL DE PÓS-GRADUAÇÃO E PESQUISA EM AMBIENTE E SOCIEDADE. Anais... Indaiatuba, 2002.

VALENTE, J. A. Aprendizagem ativa no ensino superior: a proposta da sala de aula invertida. Notícias, Brusque, 2013. Disponível em: <https://www.unifebe.edu.br/site/ docs/arquivos/noticias/2014/valente.pdf>. Acesso em: 2 set. 2015.

\section{Elisangela Pavanelo}

Possui graduação em Matemática - Licenciatura pela Universidade Estadual Paulista Júlio de Mesquita Filho (UNESP-SJRP) (2000), mestrado em Educação Matemática pela Universidade Estadual Paulista Júlio de Mesquita Filho - Rio Claro (2004) e doutorado na linha de pesquisa em Ensino de Ciências e Matemática, pela USP-SP.

elisangela.pavanelo@gmail.com

\section{José Silvério Edmundo Germano}

Graduado em Física pela Universidade Federal do Rio de Janeiro (1982), mestre em Física pelo ITA (1985) e doutor em Física pelo ITA (1992). Atualmente é professor associado do ITA e atua no desenvolvimento de projetos ligados à utilização das novas tecnologias computacionais no ensino de Física, tais como experimentos de física controlados remotamente pela internet (WEBLAB).

jsegermano@gmail.com 


\section{Priscila Lourenço Freitas Lemes}

Possui graduação em Matemática pela Universidade do Vale do Paraíba (UNIVAP), mestrado e doutorado em Física e Astronomia pela mesma universidade. Atualmente faz pós-doutorado no ITA e é professora na UNIVAP.

piprisc@gmail.com 